\section{Dealing with diabetes and pregnancy following bariatric surgery: a double-edged sword?}

Marcio C. Mancini'

$\mathrm{B}$ ariatric surgery offers substantial and sustained weight loss for most patients, with diabetes improvement or remission and also reduction in weight-related comorbidities in patients with a BMI of $35 \mathrm{~kg} / \mathrm{m}^{2}$ or more. The same benefits have not yet been established for patients with a BMI of less than $35 \mathrm{~kg} / \mathrm{m}^{2}$, since there is still limited evidence based in very few studies investigating less than one hundred patients with class I obesity. Moreover, in larger studies involving patients with higher baseline BMI such as the Swedish Obese Subjects Study, the degree of weight loss has been significantly associated with glycemic improvement, independently of the surgical procedure when stratified by weight. In spite of this, delegates of International Bariatric Surgery and Diabetes Organizations during the $2^{\text {nd }}$ Diabetes Surgery Summit in London recently approved a joint statement where surgery should be considered (consider should not have the same strength of a recommendation) for patients with type 2 diabetes and BMI between 30.0 and $34.9 \mathrm{~kg} / \mathrm{m}^{2}$ if hyperglycemia is inadequately controlled despite optimal treatment with either oral or injectable medications, including insulin; and these BMI thresholds should be reduced by $2.5 \mathrm{~kg} / \mathrm{m}^{2}$ for Asian patients (in spite of short duration of diabetes, good glycemic control and absence of insulin therapy be preoperative predictive factors of diabetes remission) (1). Recently, some cardiovascular safety trials with diabetes medications have demonstrated that these drugs offer cardiovascular protection (2); more data on hard outcomes are required to better assess not only the efficacy, but mainly the safety in very large series before endorsing the widespread indication of bariatric surgery in the subpopulation of type 2 diabetics with a BMI below $35 \mathrm{~kg} / \mathrm{m}^{2}$. A significant proportion of individuals undergoing bariatric surgery experiment weight regain, residual diabetes or diabetes relapse (i.e., around one third of initial remitters over five years do not achieve remission or have diabetes recurrence), requiring to cope with the residual diabetes and emerging obesity $(2,3)$ and also to deal with lifelong nutritional deficiencies and other potential long-term complications, such as vomiting, adhesions, strictures, gallstones, hernias, drinking problems, and small-bowel obstruction $(4,5)$. In the scope under discussion, it is essential that the diagnosis of diabetes in patients undergoing bariatric surgery be reliable and safe.

In this issue of Archives of Endocrinology and Metabolism, a study examined the adverse effects of the oral glucose tolerance test (OGTT) in 128 patients who underwent bariatric surgery, of which more than $90 \%$ were women and nearly $30 \%$ pregnant. Around two thirds of patients experienced one to four or more limiting adverse effects such as nausea, dizziness, weakness, diarrhea, tachycardia, sweating or hypoglycemia during the test. The main reasons for ordering an OGTT were presence of symptoms suggestive of hypoglycemia, pregnancy, type 2 diabetes prior to surgery, or weight gain (6). In effect, an OGTT, when performed in patients following bariatric surgery (mainly but not limited to Roux-en-Y gastric bypass) is linked to risks, and it is questionable for evaluating this cohort of patients (5).
Grupo de Obesidade e Síndrome Metabólica da Disciplina de Endocrinologia e Metabologia do Hospital das Clínicas da Faculdade de Medicina da Universidade de São Paulo (HC-FMUSP), São Paulo, SP, Brasil

Correspondence to: Marcio C. Mancini Secretaria da Disciplina de Endocrinologia e Metabologia, Av. Dr. Enéas de Carvalho Aguiar, 255, $7^{\circ}$ andar, sala 7037 05403-000 - São Paulo, SP, Brasil marcio.mancini@hc.fm.usp.br

Received on July/12/2016 Accepted on July/12/2016 DOI: 10.1590/2359-3997000000181 
In recent years, episodes of severe late postprandial hypoglycemia have been increasingly reported in gastric bypass patients. In the evaluation of these most frequently asymptomatic hypoglycemic events, fasting and post-prandial self-monitoring reflectance meter glucose determinations in the home setting, or fasting and post-prandial blood glucose concentration measurements can be requested occasionally and, if symptoms of hypoglycemia occur likewise $(5,7,8)$. These patients could also be evaluated by ambulatory continuous measuring of interstitial fluid glucose systems $(5,8)$ or a mixed meal test, preferably liquid $(7)$.

The same approach has been considered valid in the evaluation of remission and relapse of type 2 diabetes mellitus, as well as measurement of glycated hemoglobin levels $(5,8)$. Over the years, many different criteria of diabetes remission have been proposed based on maintaining fasting glucose and glycated hemoglobin levels below stricter or less strict thresholds for shorter or longer periods of time (e.g. 1, 2, 5 years postoperatively) (9). At the same time, a higher mean amplitude of glucose excursions (MAGE), a shorter time to the post-prandial peak of interstitial glucose occur (with maximum glucose levels above $200 \mathrm{mg} / \mathrm{dL}$, but with normal fasting and 2-hour post meal levels in most patients with a diabetes background before surgery), and symptoms of hypoglycemia usually were more related to the speed of glucose decrease than to the glucose level achieved, since hypoglycemic episodes were not registered on all occasions. Being so, the early post-prandial hyperglycemia may underestimate the diagnosis of diabetes if glucose measurement is done 2-hours post-meal (10).

Furthermore, glucose variability is exaggerated after Roux-en-Y gastric bypass, combining typically high and early hyperglycemic peaks and rapid glucose decreases with symptoms mimicking hypoglycemia (but on occasion being true severe hypoglycemia) (10). There are high quality evidence criteria associating postmeal and post-challenging glucose hyperglycemia and macrovascular disease, retinopathy, increased carotid intima-media thickness, oxidative stress, inflammation and endothelial dysfunction, impaired cognitive function in elderly people with type 2 diabetes, as well as decreased myocardial blood flow, and increased risk of some types of cancer. A task force of the International Diabetes Federation strongly states that post-meal hyperglycemia is harmful and should be addressed. Treatments that aim post-meal plasma glucose reduce vascular events as long as hypoglycemia is avoided. Both non-pharmacologic, such as diets with a low glycemic load, and pharmacologic therapies that preferentially lower post-meal plasma glucose targeting post-meal plasma glucose below $140 \mathrm{mg} / \mathrm{dL}(7.8 \mathrm{mmol} / \mathrm{l})(1 \mathrm{l})$.

Thus, instead of OGTT, a mixed meal test (7) and/or self-monitoring of blood glucose should be preferred for diabetes diagnosis of patients undergoing bariatric surgery since it is currently the most practical method for monitoring post meal glucose excursions, and also helps to register and to avoid hypoglycemia (11). There is evidence suggesting that employing only fasting blood glucose and glycated hemoglobin levels to diagnose diabetes in patients undergoing bariatric surgery leads to underestimation, since the glycated hemoglobin may be falsely minimized or normal in patients with higher MAGE (especially with concomitant both hypoglycemic and hyperglycemic events) (10). Although these parameters provide good specificity, the sensitivity is poor in the diagnosis of dysglycemia (12).

Nearly a third of patients studied were pregnant women who underwent bariatric surgery (6). Even if significant weight loss reduces the risk of gestational diabetes mellitus (GDM), most women remain overweight after bariatric surgery, and, thus, should be screened for GDM. The assessment can be accomplished between 24 and 28 weeks of gestation by fasting and post-prandial reflectance meter glucose determinations or blood glucose concentrations or also be evaluated by ambulatory continuous measuring of interstitial fluid glucose systems $(5,8)$. Many women who were unable to get pregnant before surgery conceive after bariatric procedures, suggesting a beneficial influence on fertility outcomes. Studies that systematically monitor adherence and nutritional status are scarce, but women in reproductive age are particularly prone for anemia, and mineral and vitamin deficits following Roux-en-Y gastric bypass. Further, patients did not adequately follow the multivitamin prescription despite careful instructions. Folic acid is considered a marker of multivitamin intake and was below the reference level in around $10 \%$ of female patients. Only 36\% (24/67) of female patients 8 years following gastric bypass had a reliable intake of multivitamin supplementation (13), increasing the possibility of vitamin deficiencies during pregnancy, thus posing risks to fetal development with long-lasting health consequences such as cognitive and motor impairment, neural tube defects, and maternal or maternal-fetal complications during pregnancy, including 
severe malnutrition requiring parenteral nutrition, and, hardly ever, death. Long-term outcomes for both the mother and her offspring are still needed (14).

Women who had undergone previous bariatric surgery (comprising more than two thousand singleton births to a mother with a history of gastric bypass, adjustable gastric band or vertical gastroplasty adjusted by maternal age, parity, early pregnancy body mass index and smoking status, educational level) have a higher rate of preterm birth than those who had not and are more likely to deliver a small-for-gestational-age infant compared with those who did not have the operation. It may be reasonable for the obstetrician to screen these pregnancies for the development of intrauterine growth restriction (15). It is not entirely clear whether this increases the risk to the fetus or has long-term consequences for his health in adulthood, but some studies suggest that maternal bariatric surgery increases the risk of metabolic disorders in the offspring. Female rats rendered obese on a high-calorie diet underwent either sleeve gastrectomy or sham surgery. Pups born to sleeve gastrectomy rats were born smaller and lighter than the offspring of control rats. Moreover, when maintained on a high fat diet after puberty, these adult offspring had a greater propensity to develop glucose intolerance, hypercholesterolemia, hepatic steatosis, and overweight or obesity than the control offspring of rats who underwent sham surgery (16). Many factors in the maternal environment can result in intrauterine growth restriction including calorie or macronutrient malnutrition, malabsorption, anemia, loss of maternal ghrelin producing cells (as a putative regulator of intrauterine growth and development, acting on GHS receptors in the placenta and offspring), thus suggesting that the maternal bariatric surgery could increase the risk of obesity, metabolic syndrome and diabetes in the next generation (14-16).

To conclude, using an OGTT in patients who underwent bariatric surgery in order to evaluate hypoglycemia, or to diagnosis of GDM, type 2 diabetes, or diabetes relapse undoubtedly, leads to rapid gastric emptying of the hypertonic glucose solution causing a dramatic shift of fluid from the intravascular component to the intestinal lumen with relative hypovolemia, increase in bowel contractility (consequences of excessive secretion of hormones vasoactive intestinal polypeptide, serotonin, and bradykinin) and, incomplete compensatory sympathetic activity, pivotal in the early dumping symptoms. The rapid small intestine transit of hyperosmolar glucose causes a massive release of the incretin hormones glucose-dependent insulinotropic polypeptide and glucagon-like peptide-1 (GLP-1) that stimulate an exaggerated insulin secretion causing reactive hypoglycemia (further aggravated by the inhibition of glucagon secretion by GLP-1) and the symptoms of late dumping symptoms (17). The evaluation of these patients by ambulatory continuous measuring of interstitial fluid glucose systems seems to be more sensitive in detecting hypoglycemia, hyperglycemia, for the diagnosis of diabetes, diabetes relapse, excessive glucose variability, and also GDM.

Funding statement: no funding statement.

Disclosure: no potential conflict of interest relevant to this article was reported.

\section{REFERENCES}

1. American Diabetes Association. Obesity Management for the Treatment of Type 2 Diabetes. Diabetes Care. 2016;39 Suppl 1:S47-51.

2. Halpern B, Cercato C, Mancini MC. Diabetes remission off medications is not a suitable endpoint for comparing bariatric/ metabolic surgery with pharmacotherapy. Diabetologia. 2016 Jun 16. [Epub ahead of print]

3. Pajecki D, Halpern A, Cercato C, Mancini M, Cleva R, Santo MA. Tratamento de curto prazo com liraglutide no reganho de peso após cirurgia bariátrica. Rev Col Bras Cir. 2013;40(3):191-5.

4. Goldfine $A B$, Patti ME. Diabetes: Bariatric surgery for T2DM cure, or remission and relapse? Nat Rev Endocrinol. 2014;10(1):8-9.

5. Mancini MC. Bariatric surgery--an update for the endocrinologist. Arq Bras Endocrinol Metabol. 2014;58(9):875-88.

6. Andrade HF, Pedrosa W, Diniz MF, Passos VM. Adverse effects during the oral glucose tolerance test in post-bariatric surgery patients. Arch Endocrinol Metab. 2016;60(4):307-13.

7. Lee CJ, Brown TT, Cheskin LJ, Choi P, Moran TH, Peterson L, et al. Effects of meal composition on postprandial incretin, glucose and insulin responses after surgical and medical weight loss. Obesity Sci Pract. 2015;1(2):104-9.

8. Xing D, Kollman C, Beck RW, TamborlaneWV, Laffel L, Buckingham BA, et al.; Juvenile Diabetes Research Foundation Continuous Glucose Monitoring Study Group. Optimal sampling intervals to assess long-term glycemic control using continuous glucose monitoring. Diabetes TechnolTher. 2011;13(3):351-8.

9. Mas-Lorenzo A, Benaiges D, Flores-Le-Roux JA, Pedro-Botet J, Ramon JM, Parri A, et al.; Obemar Group. Impact of different criteria on type 2 diabetes remission rate after bariatric surgery. Obes Surg. 2014;24(11):1881-7.

10. Hanaire H, Bertrand M, Guerci B, Anduze $Y$, Guillaume E, Ritz P. High glycemic variability assessed by continuous glucose monitoring after surgical treatment of obesity by gastric bypass. Diabetes Technol Ther. 2011;13(6):625-30.

11. International Diabetes Federation. 2011 Guideline for management of post meal glucose in diabetes. Brussels: International Diabetes Federation; 2011. Available from: http://www.idf.org. Accessed on: June $30^{\text {th }} 2016$. 
12. Cosson E, Hamo-Tchatchouang E, Banu I, Nguyen MT, Chiheb S, $\mathrm{Ba} \mathrm{H}$, et al. A large proportion of prediabetes and diabetes goes undiagnosed when only fasting plasma glucose and/or HbA1c are measured in overweight or obese patients. Diabetes Metab. 2010;36(4):312-8.

13. Dalcanale L, Oliveira CP, Faintuch J, Nogueira MA, Rondó $P$, Lima $\mathrm{VM}$, et al. Long-term nutritional outcome after gastric bypass. Obes Surg. 2010;20(2):181-7.

14. Kominiarek MA. Pregnancy after bariatric surgery. Obstet Gynecol Clin North Am. 2010;37(2):305-20.
15. Roos N, Neovius M, Cnattingius S, Lagerros YT, Sääf M, Granath $F$, et al. Perinatal outcomes after bariatric surgery: nationwide population based matched cohort study. BMJ. 2013;347:f6460.

16. Grayson BE, Schneider KM, Woods SC, Seeley RJ. Improved rodent maternal metabolism but reduced intrauterine growth after vertical sleeve gastrectomy. Sci Transl Med. 2013;5(199):199ra 112.

17. Berg P, McCallum R. Dumping syndrome: a review of the current concepts of pathophysiology, diagnosis, and treatment. Dig Dis Sci. 2016;61(1):11-8. 